\title{
Health care management
}

\author{
Sally Brailsford • Erhan Kozan • Marion S. Rauner
}

Published online: 23 June 2012

(C) Springer Science+Business Media, LLC 2012

Worldwide, the health care sector consumes a huge share of national expenditure. The continuing global financial crisis has led to decreasing health care budgets, yet at the same time there is increasing demand for better services as the population ages; therefore strategic, tactical, and operational health care management have all become critically important. For all these reasons, there is now more than ever a tremendous need for quantitative decision support systems to help policymakers plan more effectively and allocate health care resources efficiently and equitably. The primary objective of this Special Issue of the Flexible Services and Manufacturing (FSM) Journal was to reflect recent developments in quantitative techniques to improve strategic, tactical, and operational management of health care services. This special issue contains new research contributions mainly illustrated by real world examples with a special focus on the policy implications of the work.

\section{Papers in this special issue}

The seven papers published in this special issue cover a range of application areas, but share a common focus on effective and efficient use of scarce resources and improving services to patients in a real-world setting. They include new

S. Brailsford $(\bowtie)$

School of Management, University of Southampton, Southampton SO17 1BJ, UK

e-mail: S.C.Brailsford@soton.ac.uk

\section{E. Kozan}

School of Mathematical Sciences, Queensland University of Technology, GPO Box 2434, Brisbane, QLD 4001, Australia

e-mail: e.kozan@qut.edu.au

\section{S. Rauner}

School of Business and Economics, University of Vienna, Bruenner Str. 72, 1210 Vienna, Austria e-mail: marion.rauner@univie.ac.at 
methodologies such as continuous time Bayesian networks and data mining, as well as the more traditional approaches such as optimization and simulation. They cover both primary (community) and secondary (hospital) care. Two papers deal with cancer treatment, and two, with continuity of care in a primary setting. One paper presents a diagnostic and prognostic tool for use in heart disease. Overall, these seven papers illustrate the breadth of work currently being undertaken by quantitative modelers to improve the quality of patient care while at the same time utilizing precious scarce resources more efficiently.

Cancer of the colon is one of the more common, and yet most successfully treatable cancers if detected early enough. Although there are well assessed screening guidelines for colon cancer, the question is whether they are actually applied in healthcare practice. The paper by Antonelli, Baralis, Bruno, Chiusano, Mahoto and Petrigni addresses the problem of analysing diagnostic pathways followed by colon cancer patients in a public healthcare agency in Italy. By exploiting the ability of data mining techniques to extract sequences from large masses of raw data, it has been possible to reconstruct the actual diagnostic pathways accessed with larger frequency by the patients. The results show that there is a large majority of patients that followed pathways deviating from the standard guidelines.

Optimization techniques have been very successfully used for operating room scheduling and there is an extensive literature in this area. Stuart and Kozan extend this literature by addressing the disruption management and rescheduling problem of the day-to-day running of a day surgery unit. The proposed optimization model sequences both elective and non-elective patients in the online environment. The authors propose a branch and bound algorithm to solve the problem. Computational experiments are conducted illustrating its applicability to the problem of reactive scheduling in the operating theatre.

In primary care, patients are normally assigned to a specific named physician, even in a group practice. This can lead to problems with access if a physician is willing only to see his/her own patients. Balasubramanian, Muriel, and Wang investigate the impact of flexibility and capacity allocation on the performance of primary group practices. Flexibility refers to the ability of a primary care physician to see patients of other physicians. Greater flexibility implies greater levels of access, but this comes at a cost to patient-physician continuity. In this paper, the authors investigate the impact of varying levels of flexibility (fully flexible, partially flexible and dedicated) on the access performance of a primary care practice as well as on the number of diversions (losses in continuity). The flexibility here is restricted to same-day requests, where quick access to a physician outweighs the need for continuity. Prescheduled appointments are seen by the patient's own physician. The results show that a partially flexible practice-where each patient sees at most 2 physicians in the practice-performs almost as well as a fully flexible practice. Further, the capacity reserved on a given workday for same-day appointments depends both on how flexible the physicians are as well as on the overall utilization of the group practice.

Radiotherapy is a highly effective treatment for cancer, but the linear accelerator machines used to deliver this treatment are very expensive. It is therefore 
unsurprising that operations research has been widely used to optimize the utilization of this precious resource, although most of the published work focuses only on part of the process. Joustra, Kolfin, van Dijk, Koning and Bakker contribute to this literature by modeling the entire pathway of radiotherapy patients at the Academic Medical Center in Amsterdam, The Netherlands. Previous efforts, focusing primarily on managing capacity at the presumed bottleneck, namely, the linear accelerators, had not succeeded in reaching the national throughput time targets. Merely extending the operational time of the linear accelerators did not lead to significant improvements in throughput time. Joustra et al. develop a discreteevent simulation model to determine which aspects of the process are primarily responsible for preventing the radiotherapy department from meeting these throughput time targets. The model is used to search for cost-effective solutions to reduce the throughput times, to provide quantitative support to compare alternative solutions, and to show that capacity variability reduction in the multistep process can be highly effective.

Human resource planning in home care (HC) is a difficult task, especially concerning the nurse-to-patient assignment which consists of allocating each newly admitted patient to his/her key nurse, who will play a vital role in their future care. Lanzarone and Matta address the cost assignment policy for such patients. Continuity of care is desirable for the patient, since the key nurse should not keep changing. Thus, if the assignments are not to be changed for a long period, this requires an accurate workload balancing which takes into account the stochastic evolution of patient demands. Lanzarone and Matta derive a structural policy to assign a newly admitted patient while balancing the workload among the operators in a stochastic context, by minimizing the expected value of a cost function that penalizes the overtime of operators. A numerical comparison with the current practice of HC providers showed that better workload balances and cost savings can be reached by implementing the proposed policy.

In recent years Bayesian networks have gained popularity for modeling uncertainty in decision support systems, but because of their heavy computational requirements they have to date mainly been used for static, rather than dynamic systems. This is a limitation in some clinical circumstances where the evolution of a condition over time is of importance. Stella, Gatti and Luciano present a dynamic continuous time Bayesian network model for cardiogenic heart failure, one of the most common, costly, disabling and deadly medical conditions, which overcomes some of these disadvantages. The model includes both unobservable variables and clinical signs and symptoms which are obtained directly from medical investigations. The model enables clinicians to make inferences about unobservable variables, such as myocardial infarction and cardiac pump impairment, in order to make diagnostic judgements as well as prognoses about the likely occurrence of other clinical outcomes such as shock and persistent heart failure. The paper presents three scenarios which show how the model can be used for both diagnosis and prediction of serious complications.

Revenue management techniques and overbooking policies are widely used in the airline industry. In the final paper, Ratcliffe, Gilland, and Marucheck apply these ideas to outpatient clinics, and consider how clinics should allocate appointment 
capacity and use overbooking to address the growing challenges of poor appointment access and high no-shows. The problem is how to balance advance requests, which have a high no-show rate, against holding "spare" appointments for potential immediate requests which have a low no-show rate yet may not in the end be required. This numerical study shows that simple heuristics perform well compared both with the computationally complex optimal policy, and with policies from previous literature and practice.

\section{Concluding remarks}

Editing this special issue has been a truly collaborative effort, involving authors, reviewers, and ourselves the editors. We are grateful for this opportunity to showcase some of the exciting work being undertaken in this key area. We also wish to express our gratitude to the anonymous referees, for their most valuable comments and pertinent suggestions that led to improvements in both the presentation and content of the papers of this special issue. Finally, we wish to thank the authors for their contribution which made this special issue possible. 